\title{
Synthesis of first row transition metal carboxylate complexes by ring opening reactions of cyclic anhydrides
}

\author{
JUBARAJ BIKASH BARUAH \\ Department of Chemistry, Indian Institute of Technology, Guwahati 781039, India \\ e-mail: juba@iitg.ernet.in
}

\begin{abstract}
Hydrolytic and solvolytic ring opening reactions of phthalic anhydride, pyromellitic dianhydride and 2,3-pyridine dicarboxylic anhydride in the presence of various transition metal salts with or without a ancillary ligands were studied. The reactions were found to be dependent on stoichiometry of ligand and types of anhydride used. In the case of pyromellitic dianhydride selective ring opening reaction by cobalt(II) ions gave 1,3-benzene dicarboxylate derivatives, whereas use of copper(II) ions gave 1,4-benzene dicarboxylate derivatives. Preference for methanolysis was seen in the case of copper (II) promoted reactions of pyromellitic dianhydride in the presence of 1:1 metal to 1,10-phenanthroline ratio.
\end{abstract}

Keywords. Metal carboxylates; ring opening reactions; cyclic anhydrides; structural study.

\section{Introduction}

Hyrdolysis and alcoholysis of acid anhydrides are important reactions in organic chemistry. ${ }^{1-7}$ The hydrolysis of esters caused by various enzymes finds important place in biology. ${ }^{89}$ Various Lewis acid catalysts $^{10,11}$ and organocatalysts ${ }^{12}$ are used for such alcoholysis as well as for hydrolysis reactions of esters. Metal ions are known to catalyse such reactions ${ }^{13,14}$ and certain metal catalysed ring opening reactions are used as model catalyst for carboxypeptidase A. ${ }^{15}$ To make such reactions catalytic by a metal ion the intermittent metal carboxylate complex formed through ring opening reactions must easily dissociate ${ }^{13,15}$ to liberate free carboxylic acid. If they do not easily dissociate the reactions would provide means for synthesis of stable carboxylate complexes rather than causing catalytic reactions. We have studied various hydrolysis and alcoholysis reactions catalysed by metal complexes with a few cyclic anhydrides of aromatic acids and a brief account of the results are presented in this article.

\section{Discussions}

\subsection{Carboxylate complexes by ring opening reactions of phthalic anhydride}

The ring opening reactions of phthalic anhydride with different alcohols are promoted by magnesium, leads to mono ester of the corresponding alcohol under mild conditions. Such reactions when carried out with dif- ferent metal salts, these resulted in the formation of metal carboxylate complexes. ${ }^{16}$ The metallic magnesium reacts with alcohols to give alkoxides, which acts as nucleophile for ring opening reactions and maintains the $\mathrm{pH}$ suitable for formation of metal carboxylate complexes. Taking advantage of this ring opening reaction, copper and nickel complexes are prepared as depicted in scheme 1.

The phthalate esters are used for chiral separation ${ }^{17}$ of optically active alcohols or amines; and such esters are prepared via ring opening reactions of phthalic anhydrides. The reactions of phthalic anhydrides in the presence of an amino acid such as glycine also help in preparing $\mathrm{N}$-phthaloylglycinato complexes of transition metals. ${ }^{18}$ For example in situ reaction of the phthalic anhydride with glycine in presence of cobalt(II) acetate gives a hexa aqua $N$-phthaloylglycinato cobalt(II) complex (chart 1). This reaction passes through formation of $\mathrm{N}$-phthaloylglycine from the reaction of glycine with phthalic anhydride. This is confirmed by preparing the same cobalt complex from reaction of $\mathrm{N}$ phthaloylglycine with cobalt(II) acetate hexahydrate.

\subsection{Carboxylate complexes by ring opening reactions of pyromellitic dianhydride}

In general, ring opening reactions of pyromellitic dianhydride by alcohol (without any other reagent being added) leads to equimolar amount of 1,3- and 1,4dicarboxylic acids (chart 2). ${ }^{19}$ So the selectivity in these ring opening reactions are important to make useful 


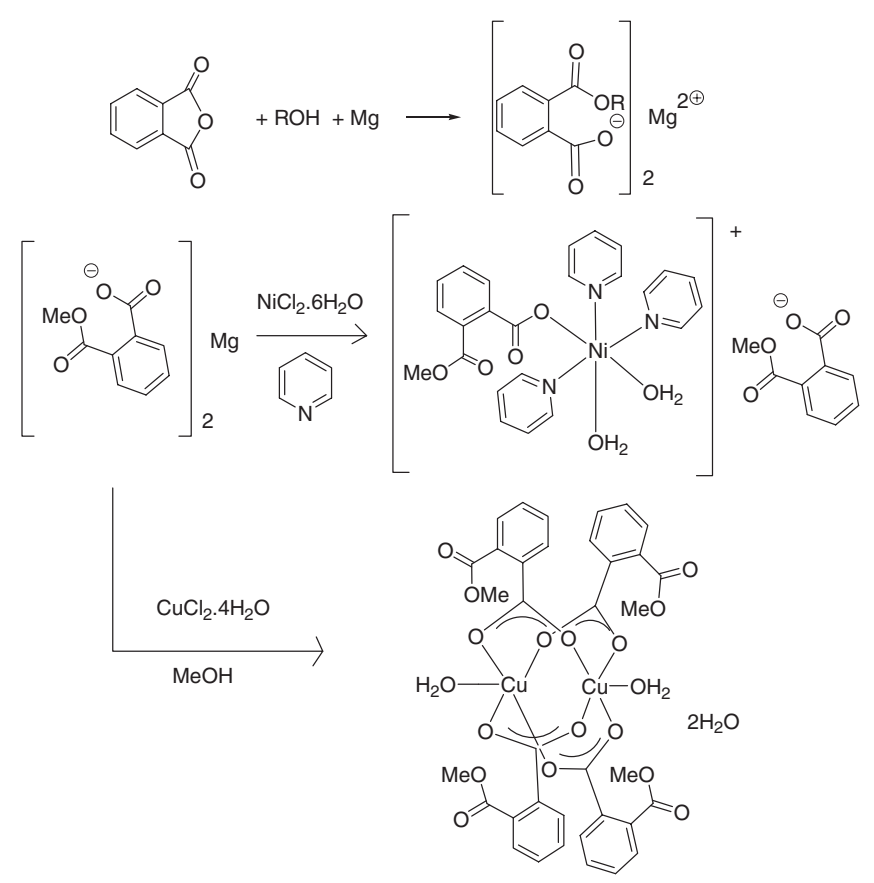

Scheme 1. Metal complexes by ring opening reaction of phthaliic anhydride.

materials. Although the use of Lewis acid in analogous ring opening reactions is very common, ${ }^{19}$ metal ion promoted reactions for such ring opening reactions to prepare inorganic complexes are limited. ${ }^{15}$

The nickel(II) acetate tetrahydrate promotes the reaction of pyromellitic dianhydride with methanol and ring opening of pyromellitic dianhydride takes place. The corresponding carboxylate complex formed during the process can be crystallised by adding bidentate nitrogen donor ligands such as 1,10-phenanthroline or 2,2'- bipyridine. ${ }^{20}$ From the reaction with 1,10 phenanthroline a metallacycle (scheme 2) containing nickel(II) ions is obtained. The product has the two ester groups at 1 and 3 positions of the aromatic ring and also the carboxylate groups bridging the metal ions are at meta positions of the ring with respect to each other. The presence of the two carboxylate groups at two positions related as meta positions provides the correct geometry to enable the two carboxylates to form the metallacycle with nickel(II) ions. The nickel (II) ions are in hexa coordinated geometry; of which two cis positions are occupied by carboxylate groups and another two cis positions are by 1,10-phenanthroline. The axial positions are occupied by a methanol and a water molecule.

However, the similar reaction of nickel(II) acetate tetrahydrate with pyromellitic dianhydride in the presence of 2,2'-bipyridine in methanol gave a mononuclear nickel(II) complex (scheme 2). This complex is totally different from the one that have 1,10 phenathroline. The 2,2'-bipyridine containing complex has non-coordinating carboxylate anions. The anionic part of the complex has the dicarboxylate dianion in which the dicarboxylates are at para position to each other. The cationic part is a complex cation of tetra-aqua (2,2'-bipyridine)nickel(II). Thus, the inorganic complexes obtained from these ring opening reactions are decided by the ancillary ligands. However, in both the cases we had other uncharacterized insoluble metal complexes due to their insolubility in common solvents and limitations in separation. But one point is clear that either of the two isomeric acid products can be characterized in pure form as nickel complex.

The reaction of pyromellitic dianhydride with zinc(II) acetate and 1,10-phenanthroline in methanol gives a crystalline coordination polymer (scheme 3 ) with a zig-zag chain like structure. The coordination polymer has zinc ions in distorted square-pyramidal geometry with five coordination number. The 1,10phenanthroline ligand and two carboxylate groups occupy two cis positions of the square pyramid. The carboxylates are bound to zinc in monodentate fashion. The fifth coordination sites of zinc(II) has water molecules. Comparison of this reaction to<smiles>COCCOC(=O)C(OC(=O)CN1C(=O)c2ccccc2C1=O)OC(=O)CN1C(=O)c2ccccc2C1=O</smiles>

Chart 1. Synthesis of $N$-phthaloylglycinato complex. 
<smiles>C=CC1OC(=O)c2cc3c(cc21)C(=O)OC3=O</smiles>

Pyromellitic dianhydride<smiles>O=C(O)c1cc(C(=O)O)c(C(=O)[OH2+])cc1C(=O)O</smiles>

cis attack of solvent<smiles>O=C(O)c1cc(C(=O)O)c(C(=O)O)cc1C(=O)O</smiles>

trans attack of solvent

Chart 2. Ring opening reaction of pyromellitic dianhydride via alcoholysis.

the similar reaction by nickel(II) together with 1,10 phenanthroline, the ring opened carboxylate at 1,3positions in the case of nickel(II) whereas the analogues reaction of zinc(II) the ring opened product has carboxylates at 1,4-positions of the ring.

When 2,2'-bipyridine is used as ligand in the reaction of zinc(II) chloride with pyromellitic dianhydride in methanol, two crystalline zinc complexes are formed. One is $\left[\mathrm{Zn}(\mathrm{bpy})_{3} \mathrm{Cl}_{2}\right] \cdot 5 \mathrm{H}_{2} \mathrm{O}$ and other is $\mathrm{Zn}$ (bpy) $\mathrm{Cl}_{2}$ (where bpy $=2,2^{\prime}$-bipyridine). The compound $\left[\mathrm{Zn}(\mathrm{bpy})_{3} \mathrm{Cl}_{2}\right] .5 \mathrm{H}_{2} \mathrm{O}$ is characterised by comparing its spectroscopic and structural data with the reported structure. ${ }^{20}$ The organic product in this reaction is found to be the 2,5-dicarbomethoxy benzene 1,4-dicarboxylic acid. From these observations it is clear that the formation of zinc(II) bipyridine complexes is favourable over the metal carboxylate complex of the corresponding dicarboxylic acid. When 2,2'bipyridine is taken at three times that of the zinc salt and reacted with pyromellitic dianhydride in methanol, the $\left[\mathrm{Zn}(\mathrm{bpy})_{3}\right] \mathrm{Cl}_{2 .} 5 \mathrm{H}_{2} \mathrm{O}$ and 2,5-dicarbomethoxy benzene 1,4-dicarboxylic acid were obtained as products. The zinc chloride is used as catalyst in various solvolytic reactions of anhydrides. ${ }^{22}$

The cobalt(II) acetate promoted reaction of pyromellitic dianhydride with methanol in the presence of either 2,2'-bipyridine or 1,10-phenanthroline are very selective. ${ }^{20}$ In each case cobalt(II) complexes having six coordinated distorted octahedral geometry with monodentate carboxylate complexes are obtained<smiles>CO[N+](=O)[O-]</smiles><smiles>O=c1oc(=O)c2cc3c(=O)oc(=O)c3cc12</smiles>



pyromellitic dianhydride

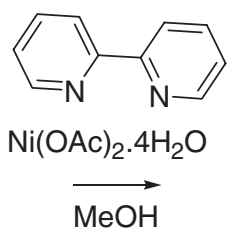

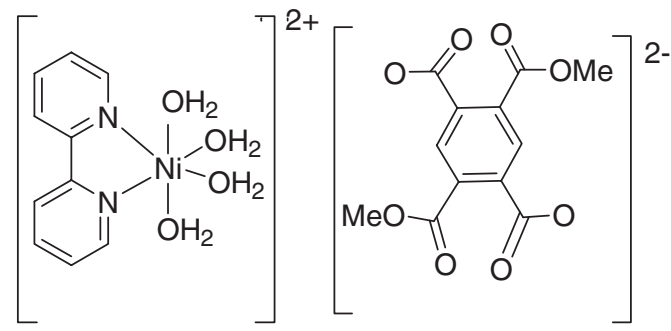

Scheme 2. A carboxylate metallacycle of nickel(II) and a carboxylate salt of nickel(II) from different ancillary ligand. 


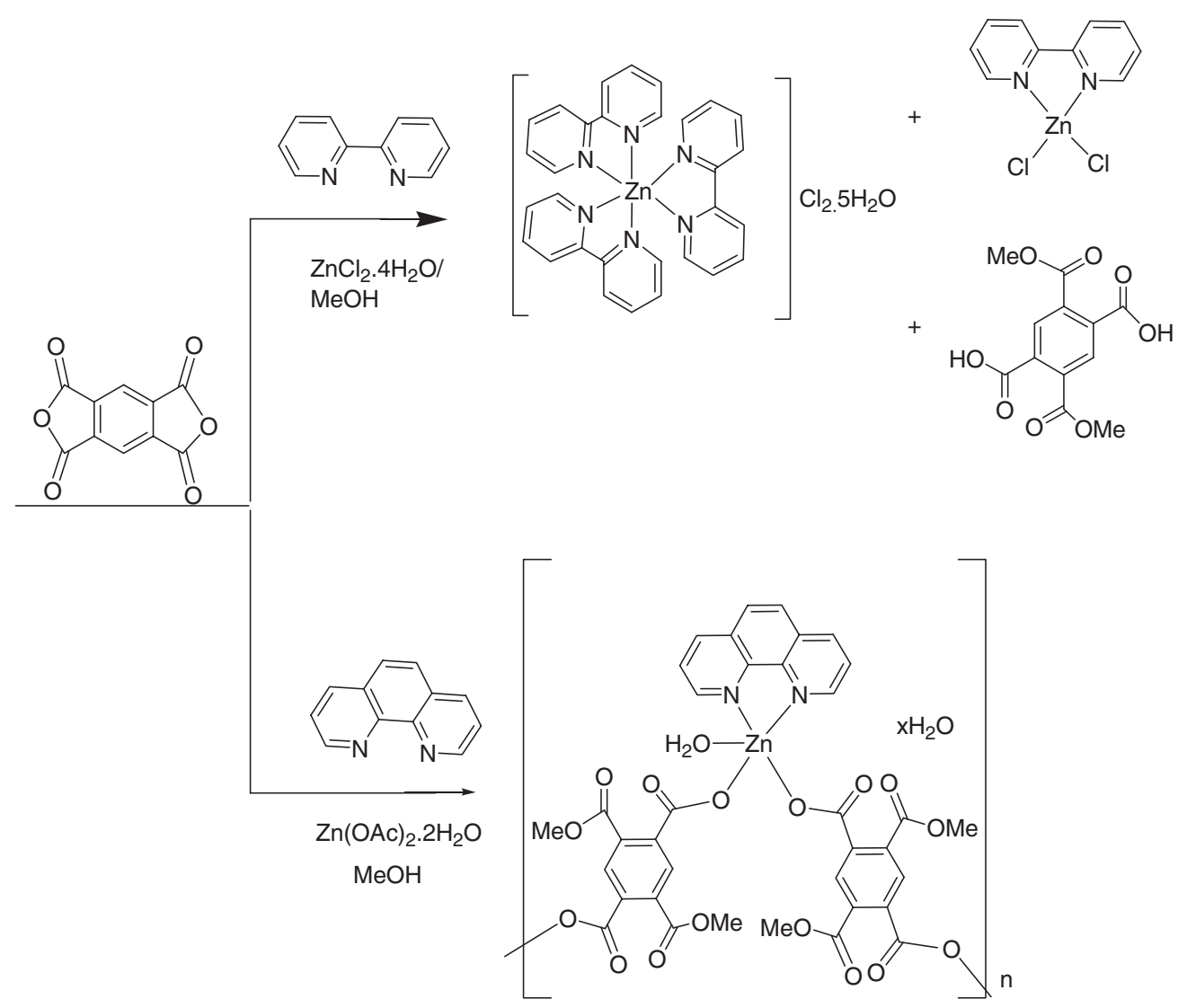

Scheme 3. The reactions of pyromellitic dianhydride with methanol promoted by zinc salts and formation of zinc complexes.

(figure 1). Selective ring opening reactions take place to generate two carboxylate anions at 1,3-positions of the aromatic ring. The carboxaylate anion at 3position of the aromatic ring remains uncoordinated. There are either two 2,2'-bipyridine ligands or one 1,10-phenanthroline ligand in the coordination sphere. The complex containing 2,2'-bipyridine has one water molecule in the coordination sphere along with

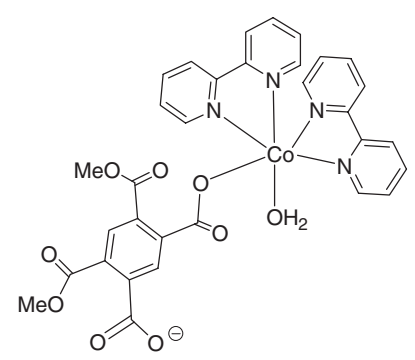

(a)



(b)
Figure 1. The cobalt complexes formed from reaction of cobalt(II) acetate tetrahydrate with pyromellitic dianhydride with methanol in the presence of (a) 2,2'-bipyridine; (b) 1,10-phenanthroline. four water of crystallisation molecules. The cobalt(II) complex having 1,10-phenanthroline, has three water ligands and a methanol molecule as solvent of crystallisation.

The reactions of pyromellitic dianhydride with methanol and copper(II) chloride as well as with copper(II) acetate in presence of bidentate aromatic heterocyclic ligands are found to be dependent on the stoichiometry of the heterocyclic ligands. ${ }^{20,21}$ The reaction with methanol carried out with 1:1 stoichiometry of metal ion to bidentate aromatic nitrogen donor ligand, methanolysis is observed; whereas same reactions with 1:2 stoichiometry of metal ion to bidentate aromatic nitrogen donor ligands resulted in hydrolysis reactions. Different products obtained from such reactions are illustrated in scheme 4 . In alcoholysis reactions we have obtained the dicarboxylate at 1 and 4-positions of aromatic ring. The products of copper(II) chloride tetrahydrate with pyromellitic dianhydride in methanol with $2,2^{\prime}$-bipyridine in $1: 1$ as well as $1: 2$ stoichiometry have identical penta coordinated distorted square pyramidal mononuclear complex cation of copper(II). The cationic part of these copper complexes 

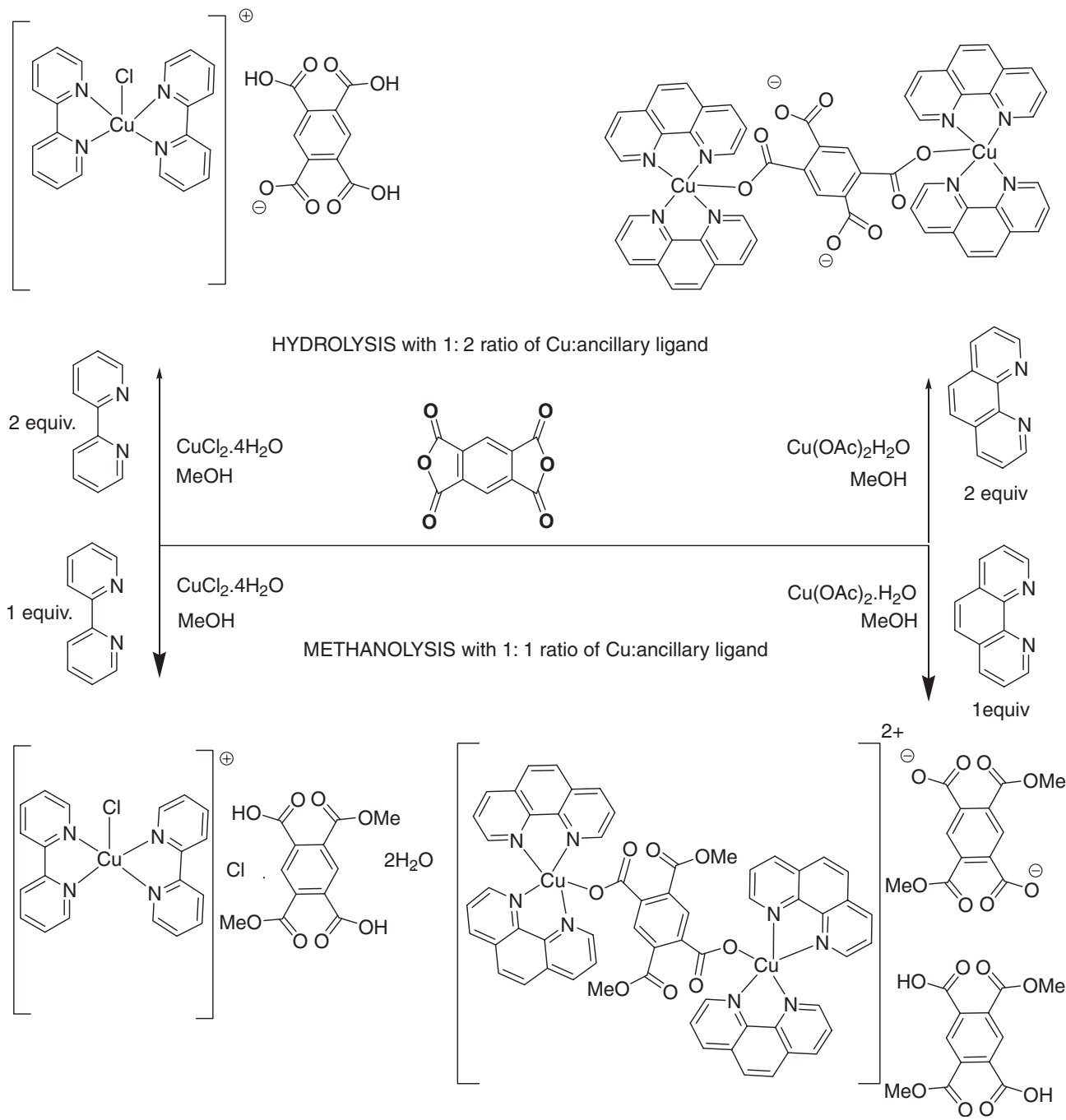

Scheme 4. Various copper(II) complexes from solvolysis reactions of pyromellitic dianhydride with different proportions of ancillary ligands.

have two 2,2'-bipyridine ligands and a chloride ligand. The difference is the anions in the two complexes. In the case of 1:1 stoichiometry anion is a chloride, the complex is obtained as a solvate with one molecule of 2,5-dicarbomethoxy benzene 1,4-dicarboxylic acid along with one water molecule. The product obtained from the reaction carried out with 1:2 ratio of copper: 2,2'-bipyridine the anion has a mono anion of 1,2,4,5-benzene tetracarboxylic acid.

The solvolysis reaction of pyromellitic dinahydride by copper(II) acetate monohydrate together with 1,10-phenanthroline (scheme 4) led to two different dinuclear complexes when the metal to 1,10 phenanthroline ratios are 1:1 and 2:1 respectively. A dinuclear complex is obtained from the reaction of 1:1 stoichiometry; this complex has two copper(II) ions bridged by a 2,5-dicarbomethoxy benzene 1,4dicarboxylato dianion. The charges of the dinuclear core in the complex is compensated by an uncoordinated 2,5-dicarbomethoxybenzene 1,4-dicarboxylato dianion. The complex has five coordinated distorted square pyramidal geometries around the copper(II) ions. Further to this, the complex is obtained in a solvated form with 2,5-dicarbomethoxy benzene 1,4dicarboxylic acid. The product from the reaction of copper(II) acetate dihydrate and 1,10-phenanthroline (1:2 ratio) with pyromellitic dianhydride in methanol is also a di-nuclear copper complex. But in this case, it is a complex of tetra-anion of 1,2,4,5-benzene tetracarboxylic acid. This complex has a square pyramidal geometry around each copper(II) and it has two 1,10phenanthroline ligands on the basal plane of the square pyramidal structure. The axial position of each square pyramid is attached to oxygen atoms of the carboxylate groups attached at 1 and 4-positions of the benzene ring of the benzene tetra carboxylate anion to bridges 
two copper ions. The bridging carboxylate groups bind to copper(II) ions in monodentate fashion. From these observations on alcoholytic as well as hydrolytic ring opening reactions, it may be said that these reactions are controlled by two major factors, one is lability of the complex formed by solvolytic reaction and second is the Lewis acidity of the metal ions.

\subsection{Carboxylate complexes by ring opening reactions 2,3-pyridine dicarboxylic anhydride}

The reaction of 2,3-pyridinedicarboxylic anhydride with first row transition metal acetates of cobalt, nickel and zinc in methanol leads to metal dicarboxylate complexes. ${ }^{21}$ These natures of the carboxylate complexes formed are found to be dependent on the metal ion and leads to hydrolytic products.

A mono-nuclear chelate complex with nickel(II) is easily formed from the reaction of nickel(II) acetate tetrahydrate with 2,3-pyridine dicarboxylic anhydride in methanol followed by treatment with pyridine (scheme 5). The complex has a distorted octahedral geometry with two pyridine ligands at axial positions. A similar chelate of cobalt(II) with water as two axial ligands is formed under ambient condition. In the case of the reaction of zinc(II) acetate tetrahydrate with 2,3-pyridine dicarboxylic anhydride in methanol and 4,4'-bipyridine gave a coordination polymer of zinc (figure 2); formed by a hydrolytic reaction. The repeated unit of the coordination polymer comprises of $\left[\left\{\mathrm{Zn}_{2}(\mathrm{~L})_{2}\right\}\right.$ bpH2 $\left.\cdot 3 \mathrm{H}_{2} \mathrm{O}\right]$ where $\mathrm{L}=$ dianion of 2,3-pyridine dicarboxylic acid and $\mathrm{bpH} 2=4,4^{\prime}$ bipyridinium dication). The coordination polymer has three water molecules as solvent of crystallization with respect to each zinc ion.



Scheme 5. Nickel and cobalt complexes from hydrolytic reactions on 2,3-pyridine dicarboxylic anhydride.

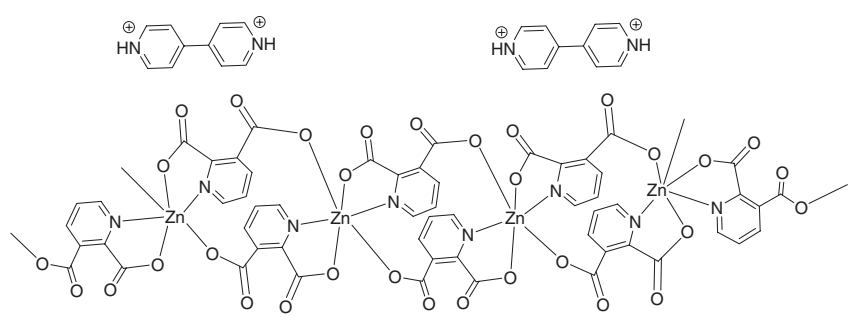

Figure 2. Coordination polymer of zinc(II) from hydrolytic reaction of 2,3-pyridine dicarboxylic anhydride with 4,4'-bipyridine.

Hydrolytic product formation in the case of 2,3pyridine dicarboxylic anhydride, suggests that the coordination effect of pyridine nitrogen atom of the ligand does not favour coordination of methanol, thus activation of methanol molecule does not take place. This leads to hydrolytic reaction. Similar explanation holds good for hydrolytic cleavage of pyromellitic dianhydride by copper(II) promoted reactions when higher amount of nitrogen donor ligands with respect to metal ion is used.

\section{Conclusions}

Hydrolysis and methanolysis reactions of different cyclic anhydrides can be achieved under mild conditions to prepare different carboxylate complexes. The selectivity on the site of the ring opening is observed in the case of cobalt(II) as well as copper(II) promoted reactions. Various complexes described in this article have wide structural variations, opens a new dimension to synthesize new complexes through ring opening reactions.

\section{Acknowledgement}

The author thanks the Department of Science and Technology, New Delhi, India for financial support.

\section{References}

1. Spivey A L and Andrews B I 2001 Angew. Chem. Int. Ed. Engl. 403131

2. Chen Y, Tian S-K and Deng L $2000 \mathrm{~J}$. Am. Chem. Soc. 1229542

3. Shimizu M, Matsukawa K and Fujisawa T 1993 Bull. Chem. Soc. Jpn. 662128

4. Kikionis S, McKee V, Markopoulos J and Lgglessi Markopoulos O 2009 Tetrahedron $\mathbf{6 5} 3711$ 
5. Takasu A, Ito M, Inai Y, Hirabayashi $\mathrm{T}$ and Nishimura Y 1999 Polym. J. 31961

6. Chemat F 2000 Tetrahedron Lett. 413855

7. Rodrignez B, Rantanen T and Bolm C 2006 Angew. Chem. Int. Ed. Engl. 456924

8. Wong C H and Whitesides G M 1994 Enzymes in synthetic organic chemistry, Oxford: Elsevier, vol 12, pp. 41-87

9. Thelsen P D and Heathcock C H 1985 J. Am. Chem. Soc. 1073731

10. Ishihara $\mathrm{K}$, Kubota $\mathrm{M}$, Kurihara $\mathrm{H}$ and Yamamoto $\mathrm{H}$ 1995 J. Am. Chem. Soc. 1174413

11. Norris J F and Rigny G W 1932 J. Am. Chem. Soc. 54 2088

12. Rho H S, Oh S H, Lee J W, Chin J and Song C E 2008 Chem. Commun. 1208

13. Wells M A and Bruice T C 1977 J. Am. Chem. Soc. 99 5341
14. Fife T H and Prystas T J 1983 J. Am. Chem. Soc. 105 1638

15. Breslow R, McClure D E, Brown R S and Eisenach J 1975 J. Am. Chem. Soc. 97194

16. Deka K, Barooah N, Sarma R J and Baruah J B 2007 J. Mol. Struct. 82744

17. Mereyala H B, Koduru S R and Cheemalpati V N 2006 Tett. Asym. 17259

18. Barooah N, Sarma R J, Batsanov A S and Baruah J B 2006 Polyhedron 2517

19. Otera J 2003 Esterification: Methods, reactions and applications, Weinheim: Wiley-VCH

20. Baruah A M, Karmakar A and Baruah J B 2007 Polyhedron 264518

21. Baruah A M, Karmakar A and Baruah J B 2007 Polyhedron 264479

22. Larrock R C (ed.) 1999 Comprehensive organic transformations 2nd ed. New York: John Wiley 